\title{
CORRIGENDUM
}

\section{Heart failure with preserved ejection fraction: insights from recent clinical researches}

\author{
Mi-Na Kim and Seong-Mi Park
}

Division of Cardiology, Korea University Anam Hospital, Seoul, Korea

Korean J Intern Med 2020;35:514-534

https://doi.org/10.3904/kjim.2020.104

Table 3. Diagnostic of HFA-PEFF [15]

\begin{tabular}{|c|c|c|c|c|}
\hline & \multicolumn{4}{|c|}{ HFA-PEFF score [15] } \\
\hline & \multicolumn{2}{|l|}{ Major } & \multicolumn{2}{|c|}{ Minor } \\
\hline & Value & Point & Value & Point \\
\hline Functional & $\begin{array}{l}\text { Septal e' }<7 \mathrm{~cm} / \mathrm{sec} \text { or lateral } \mathrm{e}^{\prime}<10 \mathrm{~cm} / \mathrm{sec} \\
\text { or } \\
\text { Averaged E/e' } \geq 15 \\
\text { or } \\
\text { TR Vmax }>2.8 \mathrm{~m} / \mathrm{sec}(\text { PASP }>35 \mathrm{mmHg})\end{array}$ & 2 & $\begin{array}{l}\text { Avergaed E/e' 9-14 } \\
\text { or } \\
\text { GLS }<16 \%\end{array}$ & 1 \\
\hline Morphological & $\begin{array}{l}\mathrm{LAVI}>34 \mathrm{~mL} / \mathrm{m}^{2} \\
\text { or } \\
\mathrm{LVMI} \geq 149 / 122 \mathrm{~g} / \mathrm{m}^{2}(\mathrm{M} / \mathrm{W}) \pm \mathrm{RWT}>0.42\end{array}$ & 2 & $\begin{array}{l}\text { LAVI } 29-34 \mathrm{~mL} / \mathrm{m}^{2} \\
\text { or } \\
\mathrm{LVMI} \geq 115 / 95 \mathrm{~m}^{2}(\mathrm{M} / \mathrm{W}) \\
\text { or } \\
\mathrm{RWT}>0.42 \\
\text { or } \\
\mathrm{LV} \text { wall thickness } \geq 12 \mathrm{~mm}\end{array}$ & 1 \\
\hline Biomarker (SR) & $\begin{array}{l}\text { NT-proBNP }>220 \mathrm{pg} / \mathrm{mL} \\
\text { or } \\
\text { BNP }>80 \mathrm{pg} / \mathrm{mL}\end{array}$ & 2 & $\begin{array}{l}\text { NT-proBNP } 125-220 \mathrm{pg} / \mathrm{mL} \\
\text { or } \\
\text { BNP }_{35}-80 \mathrm{pg} / \mathrm{mL}\end{array}$ & 1 \\
\hline$(\mathrm{AF})$ & $\begin{array}{l}\text { NT-proBNP }>660 \mathrm{pg} / \mathrm{mL} \\
\text { or } \\
\text { BNP }>240 \mathrm{pg} / \mathrm{mL}\end{array}$ & 2 & $\begin{array}{l}\text { NT-proBNP } 365-660 \mathrm{pg} / \mathrm{mL} \\
\text { or } \\
\text { BNP } 105-240 \mathrm{pg} / \mathrm{mL}\end{array}$ & 1 \\
\hline
\end{tabular}

$\geq 5$ points: heart failure with preserved ejection fraction; $2-4$ points: exercise stress test or invasive hymodynamic measurement. HFA-PEFF, heart Failure Association-PEFF; TR, tricuspid regurgitation; PASP, pulmonary artery systolic pressure; GLS, global longitudinal strain; LAVI, left atrial volume index; LVMI, left ventricular mass index; M/W, men/women; RWT, regional wall thickness; LV, left ventricular; SR, sinus rhythm; NT-proBNP, N-terminal pro-B type natriuretic peptide; BNP, B type natriuretic peptide; $\mathrm{AF}$, atrial fibrillation.

We apologize for any inconvenience that is may have caused. 